\section{An Integrated Electron Facility}

Gunnar Ingelman (Uppsala University and DESY) and Leif Jonsson (Lund University) report that the symposium Future Electron Accelerators and Free-Electron Lasers - Prospects and Opportunities in Natural Sciences (Uppsala; 25-26 April 1996) highlighted the many exciting possibilities in various sciences which are being opened up by advanced electron accelerator technology. Discussion also confirmed that an integrated electron accelerator has a great potential for interdisciplinary research. However, its effective use requires substantial technical developments within the various user communities. These would benefit from interdisciplinary collaboration (e.g., biologists collaborating with physicists to construct the new detectors needed to handle high-intensity X-rays) as well as time and funding, so the meeting ended with the planning of interdisciplinary collaborations.

An international collaboration based at the DESY laboratory in Hamburg has been carrying out for the past three years an intensive research and development programme to provide the technical basis for the construction of an $\mathrm{e}^{+} \mathrm{e}^{-}$linear collider. Two options are being pursued, namely TESLA based on superconducting 1.3 $\mathrm{GHz}$ solid $\mathrm{Nb}$ cavities and SBLC based on the well-developed $3 \mathrm{GHz}$ S-band RFcavity technology [EN 25 (1994) 104]. It has long been recognized that lowfrequency superconducting cavities have technical advantages compared to the higher frequency warm cavities. Moreover, a superconducting linac is an ideal driver for a single-pass free-electron laser (FEL).

By using extremely high purity material and new cavity processing methods, DESY recently obtained acceleration gradients of $25 \mathrm{MV} / \mathrm{m}$ at impedances $Q$ of the order of $10^{10}$ in several nine-cell $1.3 \mathrm{GHz}$ $\mathrm{Nb}$ cavities. This breakthrough means that a relatively inexpensive $500 \mathrm{GeV}-1 \mathrm{TeV}$ superconducting linear $\mathrm{e}^{+} \mathrm{e}^{-}$collider competitive with other options is feasible. Furthermore, the successful operation of very precise undulators would enable the integration of FELs based on the principle of self-amplified spontaneous emission (SASE). This would provide coherent radiation in the spectral range from the ultraviolet to the soft and even hard X-rays.

Bjorn Wiik, DESY's Director General, reported that a conceptual design for an $\mathrm{e}^{+} \mathrm{e}^{-}$linear collider facility with integrated $\mathrm{X}$-ray FELs is now in preparation. The final design report, including cost and the time schedule, will be ready by the end of 1998. Meanwhile, the TTF-FEL test facility comprising a $0.4 \mathrm{GeV}$ linear accelerator with three modules of eight 9 -cell $\mathrm{Nb}$ cavities feeding an undulator magnet is under construction. Commissioning is expected to start in $1997 / 98$ and the aim is to establish the accelerator technology and to demonstrate the principle of SASE at short wavelengths. An upgrade called VUV-FEL to $1 \mathrm{GeV}$ by adding five more cavity mod- ules is planned in order to obtain a FEL in the VUV and soft X-ray regions. A similar test facility based on the S-band technology, will start operation in 1997.

\section{High-Energy Physics}

There are powerful arguments for building a high-energy $\mathrm{e}^{+} \mathrm{e}^{-}$collider. Peter Zerwas, a theorist from DESY, described how the physics programme would be complementary to that of CERN's LHC collider, in that in addition to an exploratory potential the linear $\mathrm{e}^{+} \mathrm{e}^{-}$collider should also enable high-precision tests of the standard model. For example, the mass of the top quark could be measured with an error of only about $200 \mathrm{MeV}$ and deviations with respect to the standard model gauge-field sector are at a level of $2 \times 10^{-2}$ to $2 \times 10^{-3}$ depending on the energy.

The proposed collider is an ideal machine to look for Higgs particles in the intermediate mass range and beyond. Searches can be performed in a variety of decay channels and the properties of the Higgs particles can be thoroughly studied. Moreover, the collider is especially suited for discovering supersymmetric Higgs particles, sleptons and the non-coloured gauginos and Higgsinos. If, however, supersymmetry is not realized in Nature there might be extended gauge symmetries and/or quarks and leptons might be composites of a new level of still more fundamental matter particles.

The requirements that a detector would have to fulfill in order to pursue this ambitious programme in particle physics were described by Albrecht Wagner, DESY's Research Director. The natural starting points are detectors at CERN's LEP, and especially those at the Stanford Linear Collider Center's SLC which is the closest approximation to a linear collider. Analyses of several of the techniques used for particle tracking and calorimetry show that in order to optimize the detectors one needs to simulate the capability to measure the physics processes of interest.

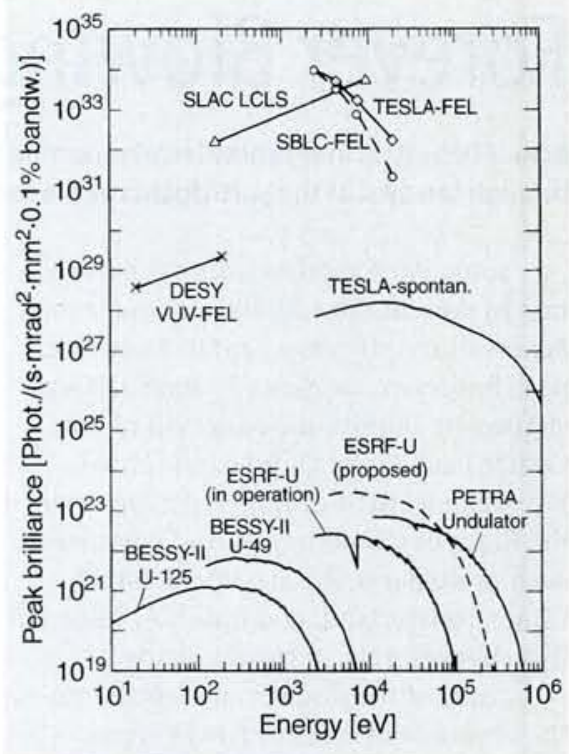

Free-electron lasers offer extremely high peak brilliance when compared with existing sources. The figure shows estimates of the peak brilliance that would be produced at FEL undulator sources integrated into full-scale $\mathrm{e}^{+} \mathrm{e}$ colliders based on SLAC (SLACLCLS), DESY S-band (SBLC-FEL) and TESLA (TESLA-FEL) technologies. They are compared with the brilliance for BESSY II (under construction) sources, for ESRF sources, for an undulator installed recently at DESY's PETRA storage ring [EN 25 (1994) 103] and for DESY's VUV-FEL upgrade of the TTFFEL test-of-principle facility now under construction.

The background radiation problem will be much more severe than at existing colliders. Components with higher radiation hardness will have to be used. Solutions will also require special masking around the beam pipe to avoid the backscattering of synchrotron radiation in quadrupole magnets, a protection system against muons from the beam halo and a collimation system to protect the machine components against beamstrahlung produced by colliding bunches. Fast-orbit feedback within the bunch train can be used to control the beam position to high precision to maintain the design luminosity. Work on the design of a detector is presently being carried out by study groups within a joint DESY - European Committee for Future Accelerators (ECFA) project. A conceptual design should be ready by the end of 1996 .

\section{High-Intensity X-Rays}

Free-electron lasers have several advantages when compared to existing conventional third-generation synchrotron radiation sources. Jochen Schneider, Director of the synchrotron radiation laboratory HASYLAB at DESY, pointed out that a SASE FEL yields a very short $(\approx 100$ 
fs) and intense ( $\approx 100 \mathrm{GW})$ burst of transversely coherent light with tuneable wavelengths in the Angström range. The extraction of beams at several points along the linac and splitting of the photon beam will allow a FEL-based radiation facility to serve many users simultaneously.

The projected peak brilliance is nearly ten orders of magnitude higher than the brilliance obtained with today's thirdgeneration sources (see figure). This, combined with the short pulse length and the high degree of coherence, offers new, unprecedented opportunities in many fields of research. Moreover, a FEL effectively combines the advantages of synchrotron radiation and conventional lasers, making it an unique tool for imaging at short wavelengths. For instance, it will be possible to obtain simultaneously direct information about the electronic and geometric structure of clusters instead of comparing theoretical models.

Schneider introduced holographic images with atomic resolution obtained using a high-intensity X-ray beams - a topic that was elaborated on by Ingolf Lindau, Director of Lund's MAX-lab, in a talk on the application of coherent X-rays in condensed-matter physics. In contrast to conventional X-ray diffraction involving elastic scattering from planes of atoms, local-source X-ray holography is based on the formation of a core-electron level vacancy in an atom by absorption of an Xray, followed by fluorescent X-ray emission at longer wavelengths. This X-ray in turn scatters elastically off nearby atoms to produce a diffraction pattern or hologram through interference between the fluorescent reference wave and the scattered wave component.

Exciting possibilities are also being opened up in X-ray photon correlation spectroscopy. This technique takes advantage of the coherence of the light. When the light is scattered from a disordered system it gives rise to a random diffraction or speckle pattern related to the exact spatial arrangement of the disorder. If the spatial arrangement of the disorder evolves with time the speckle pattern also changes and the observation of the intensity fluctuations at a single point in the speckle pattern is a direct measure of the dynamics.

\section{Atomic and Molecular Physics}

FELs also have important applications in atomic and molecular physics. Elisabeth Rachlew-Kallne from Stockholm University, in discussing some ideas and specula- tions, stressed the advantages of the pulsetime structure by pointing out that FELs enable the gap between linear and nonlinear processes to be bridged. There are, however, many technical problems to be solved, e.g., how to control the beam arrival time. The high intensity of each pulse is also a challenge for new experimental techniques.

Sune Svanberg described the Lund Laser Centre's research programme in which high-power lasers based on higherorder harmonics are being developed. Lasers of this type allow, for example, applications in time-resolved spectroscopy and photoemission studies where the emphasis is on high peak intensities for multiphoton processes at $100 \mathrm{eV}$. It was demonstrated that some of the experimental techniques can be used in conjunction with a VUV-FEL.

\section{Biology and Medicine}

Synchrotron radiation has had a major impact on research in molecular biology, where the three-dimensional structure of proteins and other biological structures are determined using X-ray crystallography. Michael Wulff from the European Synchrotron Radiation Facility in Grenoble focussed on the possibility of exploring not only structures but also dynamics using time-resolved diffraction studies at advanced X-ray sources. As an example, he described a study at liquid helium temperatures of the rebinding of photodissociated CO to myoglobelin (a globular haem protein), where the rebinding process is slow enough to allow measurements of the dynamics. He concluded that the higher intensity and resolution of an X-ray FEL would offer exciting new prospects for understanding structures and reaction dynamics in molecular biology. However, new detectors must be developed in order to fully exploit this interesting field of research.

There are many ideas and definite plans for future accelerator-based medical applications. Borje Larsson from the Institute of Medical Radiobiology and PSI in Zurich, in reviewing the potential for using radiation from synchrotrons and other sources in medical research and treatment, described how monochromatic $\mathrm{X}$-rays can be applied for medical imaging and the treatment of cancer. For example, monochromatic X-rays can be used for cancer treatment in stereotactic functional surgery, and DESY has to date successfully carried out non-invasive examinations of 39 patients using coronary angiography where iodine is injected directly into a vein in the arm. The use of X-rays for coronary angiography is based on the principle of iodine dichromography. Two images are recorded, one just below and the other just above the K-edge of iodine. Logarithmic subtraction enhances signals arising from attenuation by the iodine in the contrast medium and suppresses signals arising from attenuation by soft tissues and bone [EN 25 (1994) 102]

\section{Nuclear Physics}

There has been considerable discussion about an Electron Facility for Europe (ELFE) that would effectively represent a high-resolution microscope for measuring hadron and nuclear wave functions in nuclei [EN 21 (1990) 213]. Paul Hoyer, Director of NORDITA, and Bernard Frois from Saclay described the theoretical and experimental aspects of the nuclear physics programme foreseen for such a facility. In terms of quark and gluon dynamics, it would mean going beyond the singleparton distributions measured so far, and the study of quark dynamics could be carried further through exclusive reactions that may be identified by their final states. The measurements made to date in normal deep inelastic scattering processes are too inclusive to give information about the partons in the nucleus.

The ELFE physics programme requires an electron beam in the $15-30 \mathrm{GeV}$ energy range which is directed towards an external fixed target. The minimum energy is set by the need for a high momentum transfer to ensure a hard quark scattering process; the maximum energy implies that the distance over which the hadrons are formed is less than the radius of the target nucleus. The nucleus can then act as a detector with femtometre resolution for the hard quark scattering process.

A $30 \mathrm{~mA}$ continuous electron beam could be obtained by injecting DESY's electron beam into the existing HERA accelerator ring which would act as a beam stretcher. Detectors with $4 \pi$ coverage need to have a high-energy resolution together with fast tracking and calorimetry in order to cope with the expected luminosity of around $10^{35} \mathrm{~cm}^{-2} \mathrm{~s}^{-1}$. Recognition of exclusive final states calls for the particle identification over a broad momentum range that can be provided by counters of the RICH type. Frois concluded that an ELFE project at DESY would give Europe's nuclear physics community the opportunity to perform new and exciting physics. 